\title{
High treatment success rate for multidrug-resistant and extensively drug-resistant tuberculosis using a bedaquiline-containing treatment regimen
}

\author{
Norbert Ndjeka ${ }^{1}$, Kathryn Schnippel ${ }^{2}$, Iqbal Master ${ }^{3}$, Graeme Meintjes $^{4,5}$, \\ Gary Maartens ${ }^{6}$, Rodolfo Romero ${ }^{7}$, Xavier Padanilam ${ }^{8}$, Martin Enwerem ${ }^{9}$, \\ Sunitha Chotoo ${ }^{3}$, Nalini Singh ${ }^{3}$, Jennifer Hughes (10 ${ }^{10}$, Ebrahim Variava ${ }^{11,12}$, \\ Hannetjie Ferreira ${ }^{11}$, Julian te Riele ${ }^{13}$, Nazir Ismail ${ }^{14,15,16}$, Erika Mohr ${ }^{17}$, \\ Nonkqubela Bantubani ${ }^{18}$ and Francesca Conradie ${ }^{19}$
}

@ERSpublications

The use of bedaquiline with at least three other active drugs is associated with a high treatment success rate among preXDR-TB and XDR-TB patients in South Africa, a region of high HIV prevalence http://ow.ly/Zo7h30mii2S

Cite this article as: Ndjeka N, Schnippel K, Master I, et al. High treatment success rate for multidrugresistant and extensively drug-resistant tuberculosis using a bedaquiline-containing treatment regimen. Eur Respir J 2018; 52: 1801528 [https://doi.org/10.1183/13993003.01528-2018].

ABSTRACT South African patients with rifampicin-resistant tuberculosis (TB) and resistance to fluoroquinolones and/or injectable drugs (extensively drug-resistant (XDR) and preXDR-TB) were granted access to bedaquiline through a clinical access programme with strict inclusion and exclusion criteria.

PreXDR-TB and XDR-TB patients were treated with 24 weeks of bedaquiline within an optimised, individualised background regimen that could include levofloxacin, linezolid and clofazimine as needed. 200 patients were enrolled: 87 (43.9\%) had XDR-TB, $99(49.3 \%)$ were female and the median age was 34 years (interquartile range (IQR) 27-42). 134 (67.0\%) were living with HIV; the median CD4 ${ }^{+}$count was 281 cells $\mu \mathrm{L}^{-1}$ (IQR 130-467) and all were on antiretroviral therapy.

16 out of 200 patients $(8.0 \%)$ did not complete 6 months of bedaquiline: eight were lost to follow-up, six died, one stopped owing to side effects and one was diagnosed with drug-sensitive TB. 146 out of 200 patients (73.0\%) had favourable outcomes: 139 (69.5\%) were cured and seven (3.5\%) completed treatment. 25 patients $(12.5 \%)$ died, $20(10.0 \%)$ were lost from treatment and nine (4.5\%) had treatment failure. 22 adverse events were attributed to bedaquiline, including a QT interval corrected using the Fridericia formula $(\mathrm{QTcF})>500 \mathrm{~ms}(\mathrm{n}=5), \mathrm{QTcF}$ increase $>50 \mathrm{~ms}$ from baseline $(\mathrm{n}=11)$ and paroxysmal atrial flutter $(\mathrm{n}=1)$.

Bedaquiline added to an optimised background regimen was associated with a high rate of successful treatment outcomes for this preXDR-TB and XDR-TB cohort. 
Affiliations: ${ }^{1}$ National TB Programme, National Dept of Health, Pretoria, South Africa. ${ }^{2}$ Health Economics Unit, School of Public Health and Family Medicine, University of Cape Town, Cape Town, South Africa. ${ }^{3}$ King Dinuzulu Hospital Complex, Kwazulu Natal Dept of Health, Durban, South Africa. ${ }^{4}$ Institute of Infectious Disease and Molecular Medicine, University of Cape Town, Cape Town, South Africa. ${ }^{5}$ Dept of Medicine, University of Cape Town, Cape Town, South Africa. ${ }^{6}$ Division of Clinical Pharmacology, Dept of Medicine, University of Cape Town, Cape Town, South Africa. ${ }^{7}$ Northern Cape Dept of Health, Namakwa, South Africa. ${ }^{8}$ Sizwe Tropical Diseases Hospital, Gauteng Dept of Health, Johannesburg, South Africa. ${ }^{9}$ Amity Health Consortium, Johannesburg, South Africa. ${ }^{10}$ Desmond Tutu TB Centre, Dept of Paediatrics and Child Health, Faculty of Medicine and Health Sciences, Stellenbosch University, Cape Town, South Africa. ${ }^{11}$ Klerksdorp Tshepong Hospital, North West Dept of Health, Klerksdorp, South Africa. ${ }^{12}$ Perinatal HIV Research Unit and University of Witswatersrand, Johannesburg, South Africa. ${ }^{13}$ Brooklyn Chest Hospital, Western Cape Dept of Health, Cape Town, South Africa. ${ }^{14}$ Centre for Tuberculosis, National Institute for Communicable Diseases, National Health Laboratory Services, Johannesburg, South Africa. ${ }^{15}$ Dept of Medical Microbiology, University of Pretoria, Pretoria, South Africa. ${ }^{16}$ Dept of Internal Medicine, University of Witwatersrand, Johannesburg, South Africa. ${ }^{17}$ Médecins sans Frontières, Khayelitsha, Cape Town, South Africa. ${ }^{18}$ Medical Research Council, Durban, South Africa. ${ }^{19}$ University of the Witwatersrand, Faculty of Health Sciences, Johannesburg, South Africa.

Correspondence: Norbert Ndjeka, National TB Programme, National Department of Health, Pretoria, South Africa. E-mail: norbert.ndjekaQhealth.gov.za

\section{Introduction}

The World Health Organization (WHO) estimated that there were 600000 incident cases of rifampicin-resistant tuberculosis (RR-TB) and multidrug-resistant tuberculosis (MDR-TB) in 2016 worldwide, of which only 129689 (22\% of the global estimate) were initiated on treatment [1]. During the same period, treatment was initiated in 11192 cases of MDR- or RR-TB in South Africa ( 10\% of the global treatment cohort) and in 628 cases of MDR-TB with additional resistance to fluoroquinolones (FLQ) and second-line injectable drugs (SLI), i.e. extensively drug-resistant tuberculosis (XDR-TB) [1].

Overall poor treatment success rates, high loss to follow-up and high mortality have been the key features of RR-TB, especially for patients with XDR-TB or MDR-TB with resistance to either FLQ or SLI (preXDR-TB). There are several factors linked to poor treatment success rates for RR-TB, including the use of more toxic drugs with poorer efficacy than those used for drug-susceptible TB. In addition, until recently the treatment duration was a minimum of 18 months compared to the 6-month regimen for drug-susceptible TB. Globally, the rate of successful treatment for all TB was 83\% (2015 cohort), but the success rate for RR/MDR-TB patients (2014 cohort) was 54\% and for patients with XDR-TB only $30 \%$ [1]. For the same year, South Africa reported a success rate of $54 \%$ for RR/MDR-TB patients and $27 \%$ for XDR-TB patients [1]. Mortality was high for the 2014 cohort in South Africa; $21.7 \%$ of RR/MDR-TB patients and $42.5 \%$ of XDR-TB patients died during treatment [2]. A recent individual patient-level data meta-analysis indicated that treatment outcomes were significantly better with the use of new and repurposed drugs, including linezolid, later-generation FLQ, bedaquiline, clofazimine and carbapenems, compared to the standard treatment regimens for MDR-TB [3].

Bedaquiline was the first new anti-TB drug developed in five decades and it has a novel mechanism of action [4]. It was registered in the USA in late 2012 for MDR-TB based upon 72-week data from a phase 2 trial [5]. In the phase $2 \mathrm{~b}$ trial, treatment with 24 weeks of bedaquiline in addition to a standard background regimen resulted in increased culture conversion at 24 weeks (79\% versus 58\%) and an increased rate of cure at 120 weeks (62\% versus $44 \%$ ) compared to the background regimen with placebo [6]. However, the study also reported a statistically significant imbalance in mortality; 10 deaths (12.7\%) occurred among the 79 patients exposed to bedaquiline, but most occurred after bedaquiline was stopped, and two deaths (2.5\%) occurred in the 79 patients in the placebo arm $(p=0.02)$ [6]. In 2013, the WHO issued interim guidelines on the use of bedaquiline, indicating that it should be added to long-course regimens for RR/MDR-TB only in cases where no other effective regimen could be designed [7].

Prior to bedaquiline registration in South Africa, patients with either preXDR-TB or XDR-TB (pre/ XDR-TB) were granted access through the Bedaquiline Clinical Access Programme (BCAP), with the bedaquiline donated by Janssen Pharmaceutica [8]. This programme stopped enrolling patients around mid-March 2015 because bedaquiline was registered in October 2014 and the South African National TB Programme (SA NTP) was able to purchase the drug. We published an interim report on the data generated between March 2013 and July 2014 by the BCAP cohort [9]. This report showed that individuals responded well to bedaquiline-based regimens regardless of HIV status. It was also reported that $76 \%$ of patients who had completed $\geqslant 6$ months of bedaquiline-based treatment regimens had at least two negative TB culture results. An updated interim analysis was shared with the WHO and included in the systematic review for the 2017 update to its interim guidelines [10]. This paper reports final clinical outcomes and adverse events (AEs) of patients enrolled under BCAP. 


\section{Methods}

Inclusion and exclusion criteria

Eligible patients had a laboratory-confirmed diagnosis of pulmonary XDR-TB or preXDR-TB. Other criteria included the following: age $\geqslant 18$ years, negative pregnancy test and no history of habitual TB treatment interruption. Patients with unstable medical conditions were excluded. Patients with any of the following were also excluded: serum creatinine grade 1 or greater ( $>1.0 \times$ upper limit of normal (ULN)); lipase $>1.5 \times \mathrm{ULN}$; aspartate aminotransferase or alanine aminotransferase $\geqslant 2.0 \times \mathrm{ULN}$; and total bilirubin $>1.0 \times$ ULN. Patients with a baseline QT interval corrected using the Fridericia formula (QTcF) of $>450 \mathrm{~ms}$, clinically significant ECG abnormality at screening or a family history of prolonged QT syndrome were excluded. Patients not eligible for BCAP received standard of care individualised treatment regimens and were excluded from the analysis. No patients received delamanid while enrolled in BCAP. The need to be able to combine bedaquiline with at least three other active drugs was necessary for inclusion. The selection of these three active drugs was based on drug sensitivity tests as well as prior exposure to medicines.

\section{HIV and antiretroviral therapy regimens}

In accordance with the South African national HIV treatment guidelines, all patients living with TB and HIV are eligible for the initiation of antiretroviral therapy (ART) regardless of baseline CD4 count [11]. The standard first-line ART regimen in South Africa at the time of BCAP was tenofovir, emtricitabine and efavirenz [11]. Patients in whom a first-line regimen failed were eligible to be switched to a second-line regimen containing lopinavir and ritonavir with two appropriate nucleoside reverse transcriptase inhibitors [11]. However, efavirenz co-administration significantly reduces bedaquiline exposure [12, 13]; therefore, BCAP patients on efavirenz were switched either to nevirapine or to lopinavir and ritonavir.

\section{Pre/XDR-TB treatment and monitoring}

Bedaquiline was prescribed at a dosage of $400 \mathrm{mg}$ once daily for 2 weeks followed by $200 \mathrm{mg}$ three times a week for 22 weeks [12], alongside an individualised, optimised background regimen which included at least three second-line drugs to which the patient's TB had proven or was likely susceptible. The optimised background regimen included a combination of some or all of linezolid, clofazimine, pyrazinamide, ethambutol, high-dose isoniazid, p-aminosalicylic acid, capreomycin, kanamycin, levofloxacin, ethionamide or terizidone as per the SA NTP guidelines [14] and according to availability. Levofloxacin was used instead of moxifloxacin because it has less of an effect on the QT interval [15]. As per the interim WHO recommendations [7], QTcF intervals were measured at baseline, twice in the first month and then monthly while on bedaquiline, and liver function tests were performed at regular intervals. Serious AEs were reported as per the South African regulatory authority requirements; other AEs were indicated in the medical files and graded on a scale of mild, moderate, severe, life threatening or fatal. Sputum cultures were performed monthly. Additional laboratory monitoring (e.g. electrolytes, kidney or liver function, haemoglobin) was followed depending on the individualised regimen and ART prescribed, as per the SA NTP guidelines [14].

\section{Selection process}

Pre/XDR-TB patients were enrolled from seven approved sites across South Africa. Each site was managed by a principal investigator and a co-investigator. All investigators, pharmacists and clinical nurse practitioners working at selected sites were trained on good clinical practice. Each potential participant was presented to a National Clinical Advisory Committee consisting of eight clinicians with expertise in RR-TB. The approval of three members was required before approaching Janssen Pharmaceutica. This advisory committee, Janssen Pharmaceutica and the South African regulatory authority in turn approved the bedaquiline treatment and the optimised background regimen. The approval process took 4 weeks at the beginning of the programme, and later 2 weeks on average, during which time clinicians could initiate the optimised background regimen and optimise treatment for other comorbid conditions.

\section{Analysis and reporting}

Medical files were reviewed in June 2016 by clinicians and case record forms were captured in a longitudinal database using Research Electronic Data Capture (REDCap) hosted at the University of the Witwatersrand [16]. Medical files for patients who had not completed treatment by June 2016 were reviewed in April 2017 and outcomes updated in the database. The vital status for patients who were lost to follow-up was confirmed or updated through the national vital statistics register for those patients who had a valid South African national identity number in their medical record. We report summary statistics for patient characteristics and treatment outcomes, following the STROBE statement (www.strobestatement.org) for observational cohort studies. Poisson regression was used to test for patient or treatment characteristics associated with treatment success; incidence rate ratios (IRRs) and 95\% CI are presented. 
Multivariate analysis having adjusted for bedaquiline completion, HIV status (negative or positive) and second-line resistance category (XDR-TB, preXDR-TB with resistance to FLQ, and preXDR-TB with resistance to SLI) is also reported with adjusted IRRs (aIRRs). Statistical analysis was done in Stata version 14.2 (College Station, TX, USA).

\section{Ethical approval}

Human research ethics committee approval was secured from the University of the Witwatersrand, the University of Cape Town and Pharma-Ethics (www.pharma-ethics.co.za).

\section{Results}

\section{Patient characteristics}

From March 2013 to March 2015, 200 patients started bedaquiline in addition to a background regimen of five to eight additional anti-TB drugs at the BCAP sites; patient characteristics by HIV status are presented in table 1 . Half the participants $(n=99,49.3 \%)$ were female and the median age was 34 years (interquartile range (IQR) 27-42). For those enrolled, 87 (43.5\%) had laboratory-confirmed XDR-TB, 33 (16.5\%) had preXDR-TB (SLI) and 78 (39.0\%) had preXDR-TB (FLQ). Laboratory reports on the resistance pattern for two patients $(1.0 \%)$ were missing at the time of data extraction.

For the background regimen, clofazimine was given to 164 patients $(82.0 \%)$, levofloxacin to 166 (83.0\%) and linezolid to $128(64.0 \%)$. Among all patients, 134 (67.0\%) were living with HIV, with a median CD4 ${ }^{+}$ count of 281 cells $\mu \mathrm{L}^{-1}$ (IQR 130-467). All individuals living with HIV were on ART consisting of tenofovir, emtricitabine or lamivudine with nevirapine $(n=101,75.4 \%)$ or lopinavir and ritonavir $(n=33$, $24.6 \%)$.

\begin{tabular}{|c|c|c|c|}
\hline & HIV-negative & HIV-positive & All \\
\hline All patients & $66(33.0)$ & $134(67.0)$ & 200 \\
\hline \multicolumn{4}{|l|}{ Age years } \\
\hline Median (IQR) & $27(23-41)$ & $36(31-42)$ & $34(27-42)$ \\
\hline $18-29$ & $24(66.7)$ & 12 (33.3) & $36(18.0)$ \\
\hline $30-49$ & $31(23.0)$ & $104(77.0)$ & 135 (67.5) \\
\hline$\geqslant 50$ & 11 (37.9) & $18(62.0)$ & $29(14.5)$ \\
\hline \multicolumn{4}{|l|}{ Sex } \\
\hline Female & 31 (31.3) & $68(68.7)$ & 99 (49.3) \\
\hline Male & 35 (34.7) & $66(65.4)$ & $101(50.7)$ \\
\hline \multicolumn{4}{|l|}{ Resistance } \\
\hline preXDR-TB (FLQ) & 29 (33.3) & $58(66.7)$ & 87 (43.5) \\
\hline preXDR-TB (SLI) & 11 (33.3) & $22(66.7)$ & 33 (16.5) \\
\hline XDR-TB & $25(32.1)$ & 53 (68.0) & 78 (39.0) \\
\hline Missing & $1(50.0)$ & $1(50.0)$ & $2(1.0)$ \\
\hline \multicolumn{4}{|l|}{ Weight kg } \\
\hline Median (IQR) & 53.5 (49-65) & $55(48-62)$ & $54(48-64)$ \\
\hline$\leqslant 50$ & $25(34.7)$ & $47(65.3)$ & $72(36.0)$ \\
\hline$>50$ & 41 (32.3) & 86 (67.7) & $127(63.5)$ \\
\hline Missing & & $1(100)$ & $1(0.5)$ \\
\hline \multicolumn{4}{|l|}{ HIV status } \\
\hline Median (IQR) CD4 count cells $\mu \mathrm{L}^{-1}$ & $\mathrm{~N} / \mathrm{A}$ & $281(130-467)$ & $\mathrm{N} / \mathrm{A}$ \\
\hline ART & $\mathrm{N} / \mathrm{A}$ & $134(100)$ & $\mathrm{N} / \mathrm{A}$ \\
\hline Viral load $>1000$ copies & $\mathrm{N} / \mathrm{A}$ & $24(17.9)$ & $\mathrm{N} / \mathrm{A}$ \\
\hline \multicolumn{4}{|l|}{ Province } \\
\hline Eastern Cape & $3(50.0)$ & $3(50.0)$ & 6 (3) \\
\hline Gauteng & $6(24.0)$ & 19 (76.0) & 25 (12.5) \\
\hline KwaZulu Natal & $13(19.7)$ & 53 (80.3) & 66 (33) \\
\hline North West & $6(17.1)$ & $29(82.9)$ & 35 (17.5) \\
\hline Western Cape & 38 (55.9) & $30(44.1)$ & $68(34)$ \\
\hline
\end{tabular}

Data are presented as $\mathrm{n}$ ( $\%$ of row), unless otherwise stated. IQR: interquartile range; preXDR-TB: pre-extensively drug-resistant tuberculosis; FLQ: fluoroquinolone resistant; SLI: second-line injectable drug resistant; XDR-TB: extensively drug-resistant tuberculosis; ART: antiretroviral therapy; N/A: not applicable. 
Treatment outcomes

Among the 200 BCAP patients, 146 (73.0\%) had a favourable outcome (table 2); 139 (69.5\%) were cured and seven (3.5\%) successfully completed treatment. Among the 87 patients with the most extensive resistance (XDR-TB), 70 (80.5\%) had a successful outcome.

22 patients experienced at least one treatment interruption for bedaquiline; 16 out of 200 patients $(8.0 \%)$ did not complete 24 weeks of bedaquiline: of these, eight (50.0\%) were lost from care, six (37.5\%) died, one (6.3\%) stopped for side effects other than QTcF prolongation and one $(6.3 \%)$ was found to have drug-sensitive TB. Among the 184 BCAP patients who completed the 24 weeks of bedaquiline, $145(78.8 \%)$ had a successful outcome.

During the 18-24 months of follow-up after bedaquiline initiation, 25 patients (12.5\%) died, 20 (10.0\%) were lost from treatment and nine (4.5\%) experienced treatment failure with continued culture-positive sputa. Subsequent to discharge from BCAP, two $(22.2 \%)$ of the nine patients in whom treatment had failed died.

In unadjusted Poisson regression, completion of bedaquiline was associated with an IRR of 1.05 for success (95\% CI 1.03-1.08). When adjusted for HIV status (negative or positive) and resistance status (XDR-TB, preXDR-TB (FLQ) or preXDR-TB (SLI)), the IRR (95\% CI) was unchanged (table 3). In both univariate and adjusted regression, patients with preXDR-TB with FLQ resistance were statistically significantly less likely to have a successful treatment outcome (aIRR 0.81, 95\% CI 0.67-0.99).

\section{Reported AEs}

At baseline (initiation of bedaquiline), the median QTcF ( $n=194)$ was $403 \mathrm{~ms}$ (IQR 389-422). For the 153 patients with a reported QTcF at the end of 24 weeks of bedaquiline, the median increase from baseline was $11 \mathrm{~ms}$ (IQR -6-27). In total, 10 patients experienced 15 AEs related to QTcF prolongation.

\section{TABLE 2 Treatment outcomes by patient and treatment characteristics}

\begin{tabular}{|c|c|c|c|c|c|}
\hline & Subjects $n$ & $\begin{array}{c}\text { Successful } \\
\text { (cure or completion) }\end{array}$ & Died & Lost from treatment & Treatment failed \\
\hline All patients & & $146(73.0)$ & 25 (12.5) & $20(10.0)$ & $9(4.5)$ \\
\hline $18-29$ & 36 & $27(75.0)$ & $3(8.3)$ & $4(11.1)$ & $2(5.6)$ \\
\hline $30-49$ & 135 & $102(75.6)$ & $17(12.6)$ & 12 (8.9) & $4(3.0)$ \\
\hline$\geqslant 50$ & 29 & $17(58.6)$ & $5(17.2)$ & $4(13.8)$ & $3(10.3)$ \\
\hline Male & 101 & $66(65.4)$ & 15 (14.9) & 15 (14.9) & $5(5.0)$ \\
\hline \multicolumn{6}{|l|}{ Resistance } \\
\hline preXDR-TB (FLQ) & 78 & $50(64.1)$ & 15 (19.2) & 8 (10.3) & $5(6.4)$ \\
\hline preXDR-TB (SLI) & 33 & $25(75.8)$ & $2(6.1)$ & $6(18.2)$ & $0(0.0)$ \\
\hline XDR-TB & 87 & 70 (80.5) & $8(9.2)$ & $5(5.7)$ & $4(4.6)$ \\
\hline Missing resistance report & 2 & $1(50.0)$ & 0 & $1(50.0)$ & 0 \\
\hline \multicolumn{6}{|l|}{ HIV status } \\
\hline Negative & 66 & $44(66.7)$ & $6(9.1)$ & 12 (18.2) & $4(6.1)$ \\
\hline Positive & 134 & $102(76.1)$ & $19(14.2)$ & $8(6.0)$ & $5(3.7)$ \\
\hline HIV viral load $>1000$ copies & 24 & 14 (58.3) & $4(16.7)$ & $4(16.7)$ & $2(8.3)$ \\
\hline \multicolumn{6}{|l|}{ Bedaquiline } \\
\hline Completed 24 weeks & 184 & $145(78.8)$ & $15(8.2)$ & 15 (8.2) & $9(4.9)$ \\
\hline Incomplete & 16 & $1(6.3)$ & $10(62.5)$ & 5 (31.3) & $0(0)$ \\
\hline \multicolumn{6}{|c|}{ Other drugs included in the background regimen } \\
\hline Clofazimine & 164 & $120(73.2)$ & $19(11.6)$ & $18(11.0)$ & $7(4.3)$ \\
\hline Kanamycin & 40 & 32 (65.3) & $10(20.4)$ & 4 (8.2) & $3(6.1)$ \\
\hline Levofloxacin & 166 & 122 (73.5) & $20(12.1)$ & $16(9.6)$ & $8(4.8)$ \\
\hline Linezolid & 128 & $98(76.6)$ & 14 (10.9) & 8 (6.3) & $8(6.3)$ \\
\hline
\end{tabular}

Data are presented as $\mathrm{n}$ (\% of row), unless otherwise stated. preXDR-TB: pre-extensively drug-resistant tuberculosis; FLQ: fluoroquinolone resistant; SLI: second-line injectable drug resistant; XDR-TB: extensively drug-resistant tuberculosis. 


\begin{tabular}{|c|c|c|}
\hline & IRR $(95 \%$ CI) & $\operatorname{alRR}(95 \% \mathrm{Cl})^{\#}$ \\
\hline \multicolumn{3}{|l|}{ Age years } \\
\hline $18-29$ & 0.99 (0.80-1.23) & $1.03(0.82-1.30)$ \\
\hline $30-49$ & Reference & Reference \\
\hline$\geqslant 50$ & $0.78(0.56-1.07)$ & $0.80(0.59-1.09)$ \\
\hline \multicolumn{3}{|l|}{ Sex } \\
\hline Female & Reference & Reference \\
\hline Male & $0.81(0.68-0.96)$ & $0.83(0.71-0.98)$ \\
\hline \multicolumn{3}{|l|}{ Resistance } \\
\hline preXDR-TB (FLQ) & $0.80(0.65-0.97)$ & $0.81(0.67-0.99)$ \\
\hline preXDR-TB (SLI) & $0.94(0.76-1.17)$ & $0.97(0.80-1.17)$ \\
\hline XDR-TB & Reference & Reference \\
\hline Missing resistance report & $0.62(0.50-2.50)$ & $0.68(0.20-2.32)$ \\
\hline \multicolumn{3}{|l|}{ Weight kg } \\
\hline$\leqslant 50$ & $1.05(0.88-1.24)$ & $1.02(0.86-1.20)$ \\
\hline$>50$ & Reference & Reference \\
\hline \multicolumn{3}{|l|}{ HIV status } \\
\hline Negative & Reference & Reference \\
\hline Positive & 1.14 (0.94-1.39) & $1.18(0.98-1.42)$ \\
\hline HIV viral load $>1000$ copies & $0.78(0.55-1.10)$ & $0.87(0.62-1.20)$ \\
\hline \multicolumn{3}{|l|}{ Bedaquiline } \\
\hline Completed 24 weeks & $1.05(1.03-1.08)$ & $1.05(1.03-1.08)$ \\
\hline Incomplete & Reference & Reference \\
\hline \multicolumn{3}{|c|}{ Other drugs included in the background regimen } \\
\hline Clofazimine & $1.01(0.81-1.27)$ & $0.94(0.76-1.16)$ \\
\hline Kanamycin & $0.87(0.69-1.08)$ & $0.96(0.74-1.24)$ \\
\hline Levofloxacin & $1.04(0.82-1.32)$ & $0.99(0.79-1.24)$ \\
\hline Linezolid & $1.15(0.95-1.39)$ & $1.14(0.94-1.39)$ \\
\hline
\end{tabular}

Study investigators recorded a total of 603 AEs for 171 of 200 patients (85.5\%). Nearly all AEs were assessed by clinicians as being mild or moderate ( $n=507$ AEs, 84.1\%). Of the 603 AEs, investigators attributed $19(3.2 \%)$ to bedaquiline: increased QTcF to $>500 \mathrm{~ms}$ ( $\mathrm{n}=5$ of $19 \mathrm{AEs}, 26.3 \%)$, QTcF increase $>50 \mathrm{~ms}$ from baseline but $<500 \mathrm{~ms}$ ( $\mathrm{n}=8$ of $19,42.1 \%)$, paroxysmal atrial flutter $(\mathrm{n}=1$ of $19,5.3 \%)$ and other mild $\mathrm{AE}$ ( $\mathrm{n}=5$ of $19,26.3 \%)$.

87 AEs were reported as serious (death, life threatening, hospitalisation, significant disability, congenital anomaly, medically significant); all were graded as severe, life threatening or fatal and they occurred in one third of patients ( $n=64$ of $200,32.0 \%$ ). Among the severe AE, four (4.6\%) were attributed to bedaquiline (QTcF increase $>500 \mathrm{~ms}$ ). The most common severe AEsz were anaemia ( $\mathrm{n}=12$ of $87,13.6 \%)$, peripheral neuropathy $(n=9$ of $87,10.2 \%)$ and hearing loss or ototoxicity $(n=7$ of $87,8.0 \%)$. Severe AE were most frequently attributed to linezolid ( $\mathrm{n}=23$ of $87,26.4 \%$ ), kanamycin ( $\mathrm{n}=11$ of $87,11.4 \%)$ and terizidone ( $\mathrm{n}=8$ of $87,9.1 \%)$.

\section{Discussion}

In 2012, the SA NTP launched the BCAP in order to improve patient outcomes while simultaneously assessing the effectiveness and safety in routine settings of adding bedaquiline to individualised treatment regimens for persons with preXDR-TB and XDR-TB. This cohort of patients was one of the first to receive bedaquiline outside of a clinical trial and 134 out of 200 patients (67\%) were living with HIV. Clinicians at the sites selected patients who had few if any other treatment options. Despite this, we found that 146 out of $200(73.0 \%)$ had favourable outcomes and only 25 out of $200(12.5 \%)$ died. Tolerability of the bedaquiline-containing regimens was remarkable in our context: only 16 out of 200 patients (8.0\%) did not complete 6 months of bedaquiline. No deaths were attributed to bedaquiline.

It is encouraging that the final cohort results are consistent with the published interim results $[9,10]$. Based on the clinical trials, interim results and the experiences of clinicians working with bedaquiline in 
South Africa, the South African regulatory authority approved bedaquiline for the treatment of RR/ MDR-TB at the end of 2014. Following this approval, the SA NTP and the BCAP Clinical Advisory Committee developed guidelines for the use of bedaquiline for patients with pre/XDR-TB and for MDR/ RR-TB patients for whom an effective regimen could not otherwise be constructed [17]. By June 2018, more than 15000 patients had been initiated on bedaquiline through the SA NTP. Among the cohort of XDR-TB patients starting treatment between July 2014 and March 2016, bedaquiline-containing regimens were associated with a reduction in the risk of all-cause mortality (hazard ratio $0.26,95 \%$ CI $0.18-0.38$ ) compared with non-bedaquiline regimens [18].

The final results of BCAP, despite the high rates of second-line drug resistance and HIV infection, are also consistent with reports from other contexts [19]. At 120 weeks, the open-label trial TMC207-C209 reported 16 deaths out of 233 enrolled patients (6.9\%), none of which were considered to be related to bedaquiline [20]. Use of bedaquiline was associated with a twofold improvement in treatment success (adjusted OR 2.0, 95\% CI 1.4-2.9) in an individual patient-level data meta-analysis [3]. A multi-centre study reported a success rate for treatment of XDR-TB in patients receiving bedaquiline of $72.6 \%-80.4 \%$ [21].

Prior to the introduction of bedaquiline, the 2012 XDR-TB cohort $(n=581)$ showed a treatment success rate of $19 \%$ and death rate of $47 \%$ [2]. The 2015 XDR-TB cohort $(n=781)$ had a treatment success rate of $49 \%$ and death rate of $28 \%$ [2]. We accessed the vital registration data to update records of deaths on the BCAP as well as the $2015 \mathrm{XDR}-\mathrm{TB}$ cohort.

An analysis of this cohort showed that providing a bedaquiline-based regimen to $65 \%$ of individuals in the XDR-TB cohort of 2015 helped to significantly increase overall treatment success rate and significantly decrease death rate. Following the phase $2 b$ trial results, the safety and tolerability of bedaquiline-containing regimens have been questioned and this has contributed to the slow uptake of bedaquiline. Meanwhile, the global treatment success rates for MDR-TB and XDR-TB have been stagnant and poor. Recently, several studies have shown that bedaquiline is well tolerated and effective with a good safety profile [22, 23]; hence, there has been a call for widespread use of bedaquiline-containing regimens [24], with background regimens including linezolid, clofazimine and levofloxacin. Gatifloxacin has been recommended and on the list of FLQs in all guidelines of the WHO [25] and has shown very good results in short-course MDR-TB treatment [26]. Unfortunately, gatifloxacin is not widely available. While moxifloxacin could be used in this regimen, the concern about the increase in QT interval remains [15]. Gatifloxacin or moxifloxacin could be more effective than levofloxacin, although that has to be further investigated.

In the global TB Alliance trial NIX-TB, patients with XDR-TB, MDR-TB treatment intolerance or MDR-TB treatment failure were started on a combination of bedaquiline, pretomanid and linezolid. The last published results from this trial were in February 2017 [27]; in a personal communication with the TB Alliance, the high rate of success has remained $>80 \%$ with mortality $<10 \%$.

\section{Limitations}

The observational and programmatic design of this study is a limitation, because there was no control arm and patients were seen in implementation rather than study settings.

Based on early phase 2 data from the trial TMC207 [28], the US Food and Drug Administration approved bedaquiline in December 2012. This was under accelerated approval based on the time to sputum culture conversion. Continued approval for this indication is contingent upon the verification and a description of clinical benefit in confirmatory trials. STREAM Stage 2 has been designed as the phase 3 trial for bedaquiline. This is at present a three-arm study comparing the WHO-approved 9-11-month treatment regimen for MDR-TB to two bedaquiline-containing regimens. The first of these is a 9-month injection-free regimen and the second is a 6-month injectable-drug-containing regimen. This is a multi-centre trial being conducted in South Africa, Ethiopia, Uganda, Mongolia and India. Enrolment has been slower than expected and results are expected in late 2021. While randomised controlled clinical trials remain the highest level of medical evidence and still have to be performed, the results of this cohort are reassuring.

\section{Conclusions}

This cohort with resistance to FLQ and/or SLIs had a high proportion of final successful treatment outcomes when treated with bedaquiline-containing regimens. While AEs occurred, most were indicated as probably attributed to drugs in the background regimen and not bedaquiline. These encouraging results supported the SA National Department of Health's bold decision to remove the injectable agent from the MDR-TB treatment regimen and replace it with bedaquiline. These results were included in the analysis of evidence that informed the latest recommendation with regard to the use of bedaquiline in MDR-TB and XDR-TB. The WHO recently updated their drug-resistant TB medicines classification: bedaquiline has 
moved to group A, which comprises the most potent medicines to treat drug-resistant $\mathrm{TB}$, alongside linezolid and the latest-generation FLQs; kanamycin is no longer recommended [29]. Finally, it is not the addition of a single medication to a regimen for the treatment of MDR-TB or XDR-TB that will prove to be a game changer. Instead, new regimens are needed that combine many of the new and repurposed agents, including linezolid, carbapenem and other companion drugs, if we are to decrease the morbidity and mortality associated with RR-TB.

Acknowledgements: Thank you to the staff and patients at all the BCAP sites included in this final analysis and to clinicians, staff and patients at all the newly participating BCAP sites. Special thanks to Cynthia Firnhaber, Liesl Page-Shipp, Nkateko Mkhondo and Salome Charalambous for file reviews and Mathapelo Mafohla for coordinating final data updating. Thanks to Anja Reuter for providing useful comments to the manuscript. Thanks to Yogan Pillay, David Mametja, Anban Pillay, Gavin Steel and Mandisa Hela for supporting this project from its inception. Our provincial DR-TB managers and facilities staff are also acknowledged for their contributions.

Author contributions: N. Ndjeka, F. Conradie, J. Hughes and N. Bantubani designed the study and obtained approval for BCAP. N. Ndjeka, K. Schnippel and F. Conradie drafted the manuscript; G. Maartens, J. Hughes and G. Meintjes revised the manuscript. K. Schnippel, F. Conradie and N. Ndjeka analysed the data. SA BCAP investigators provided site leadership, medical care and collected data. All authors reviewed and approved the manuscript for submission.

Conflict of interest: N. Ndjeka reports non-financial support (donation of bedaquiline) from Janssen Pharmaceutica, during the conduct of the study; and Janssen Pharmaceutica provided support to the SA TB Programme: funding for training, provision of ECG machines and hearing tests machines. N Ndjeka is an official within the South African Department of Health; his responsibilities include recommending guidelines for drug-resistant $\mathrm{TB}$ treatment. K. Schnippel has nothing to disclose. I. Master has nothing to disclose. G. Meintjes has nothing to disclose. G. Maartens has nothing to disclose. R. Romero has nothing to disclose. X. Padanilam has nothing to disclose. M. Enwerem has nothing to disclose. S. Chotoo has nothing to disclose. N. Singh has nothing to disclose. J. Hughes has nothing to disclose. E. Variava has nothing to disclose. H. Ferreira has nothing to disclose. J. te Riele has nothing to disclose. N. Ismail has nothing to disclose. E. Mohr has nothing to disclose. N. Bantubani has nothing to disclose. F. Conradie reports sponsorship for travel and registration for conferences from Janssen Pharmacuetica, outside the submitted work.

Support statement: Bedaquiline under BCAP was provided by Janssen Pharmaceutical. Janssen did not have any role in the collection, analysis or interpretation of data; in writing the report; or in the decision to submit the paper for publication. WHO South Africa provided funding for the final data updating. Funding information for this article has been deposited with the Crossref Funder Registry.

\section{References}

World Health Organization. Global Tuberculosis Report. Geneva, World Health Organization, 2017. South African Department of Health. Electronic Drug Resistant TB Case Register. https://edrweb.net Collaborative Group for the Meta-Analysis of Individual Patient Data in MDR-TB Treatment-2017. Treatment correlates of successful outcomes in pulmonary multidrug-resistant tuberculosis: an individual patient data meta-analysis. Lancet 2018; 392: 821-834.

4 Diacon AH, Pym AS, Grobusch M, et al. The diarylquinoline TMC207 for multidrug-resistant tuberculosis. N Engl J Med 2009; 360: 2397-2405.

5 Cox E, Laessig K. FDA approval of bedaquiline - the benefit-risk balance for drug-resistant tuberculosis. $N$ Engl J Med 2014; 371: 689-691.

6 Diacon AH, Pym AS, Grobusch MP, et al. Multidrug-resistant tuberculosis and culture conversion with bedaquiline. $N$ Engl J Med 2014; 371: 723-732.

7 World Health Organization. The Use of Bedaquiline in the Treatment of Multi-Drug Resistant Tuberculosis: Interim Policy Guidance. Geneva, World Health Organization, 2013; p. 64.

8 Conradie F, Meintjes G, Hughes J, et al. Clinical Access to Bedaquiline Programme for the treatment of drug-resistant tuberculosis. South African Med J 2014; 104: 164-166.

9 Ndjeka N, Conradie F, Schnippel K, et al. Treatment of drug-resistant tuberculosis with bedaquiline in a high HIV prevalence setting: an interim cohort analysis. Int J Tuberc Lung Dis 2015; 19: 979-985.

10 World Health Organization. Report of the Guideline Development Group Meeting on the Use of Bedaquiline in the Treatment of Multidrug-Resistant Tuberculosis, a Review of Available Evidence. Geneva, World Health Organization, 2017.

11 South African National Department of Health. The South African Antiretroviral Treatment Guidelines. Pretoria, Department of Health, 2013.

12 Sirturo [prescribing information]. Titusville, NJ, Janssen Therapeutics, 2012; p. 25.

13 Svensson EM, Aweeka FT, Park JG, et al. Model-based estimates of the effects of efavirenz on bedaquiline pharmacokinetics and suggested dose adjustments for patients coinfected with HIV and tuberculosis. Antimicrob Agents Chemother 2013; 57: 2780-2787.

14 Directorate Drug-Resistant TB, TB \& HIV. Management of Drug-Resistant TB: Policy Guidelines. Pretoria, National Department of Health, 2013.

15 Noel G. Effects of three fluoroquinolones on QT interval in healthy adults after single doses. Clin Pharmacol Ther 2003; 73: 292-303.

16 Harris PA, Taylor R, Thielke R, et al. Research electronic data capture (REDCap)-a metadata-driven methodology and workflow process for providing translational research informatics support. J Biomed Inform 2009; 42: 377-381.

17 Directorate Drug-Resistant TB, TB \& HIV. Introduction of new drugs, drug regimens and management for drug-resistant TB in South Africa: policy framework. 1.1. Pretoria, National Department of Health, 2015. 
18 Schnippel K, Ndjeka N, Maartens G, et al. Effect of bedaquiline on mortality in South African patients with drug-resistant tuberculosis: a retrospective cohort study. Lancet Respir Med 2018; 6: 699-706.

19 Pontali E, Sotgiu G, D’Ambrosio L, et al. Bedaquiline and multidrug-resistant tuberculosis: a systematic and critical analysis of the evidence. Eur Respir J 2016; 47: 394-402.

20 Pym AS, Diacon AH, Tang SJ, et al. Bedaquiline in the treatment of multidrug- and extensively drug resistant tuberculosis. Eur Respir J 2016; 47: 564-574.

21 Borisov SE, Dheda K, Enwerem M, et al. Effectiveness and safety of bedaquiline-containing regimens in the treatment of MDR- and XDR-TB: a multicentre study. Eur Respir J 2017; 49: 1700387.

22 Pontali E, Sotgiu G, Tiberi S, et al. Cardiac safety of bedaquiline: a systematic and critical analysis of the evidence. Eur Respir J 2017; 50: 1701462.

23 Pontali E, Ambrosio LD, Centis R, et al. Multidrug-resistant tuberculosis and beyond: an updated analysis of the current evidence on bedaquiline. Eur Respir J 2017; 49: 1700146.

24 Gandhi NR, Brust JCM, Shah N. A new era for treatment of drug-resistant tuberculosis. Eur Respir J 2018; 52: 1801350 .

25 World Health Organization. Companion Handbook to the Who Guidelines for the Programmatic Management of Drug-Resistant Tuberculosis. Geneva, World Health Organization, 2014.

26 Van Deun A, Kya A, Maug J, et al. Short, highly effective, and inexpensive standardized treatment of multidrugresistant tuberculosis. Am J Crit Care Med 2010; 82: 684-692.

27 Conradie F, Diacon AH, Everitt D, et al. 2017. The NIX-TB trial of pretomanid, bedaquiline, and linezolid to treat XDR-TB. www.croiwebcasts.org/p/2017croi/croi33483 Date last accessed: November 14, 2018. Date last updated: February 15, 2017.

28 Diacon AH, Donald PR, Pym A. Randomized pilot trial of eight weeks of bedaquiline (TMC207) treatment for multidrug-resistant tuberculosis: long-term outcome, tolerability, and effect on emergence of drug resistance. Antimicrob Agents Chemother 2012; 56: 3271-3276.

29 World Health Organization. Rapid Communication: Key changes to Treatment of Multidrug- and Rifampicin-resistant Tuberculosis. Geneva, World Health Organization, 2018. 